\title{
Long term-follow-up multicenter feasibility study of ICG fluorescence-navigated sentinel node biopsy in oral cancer
}

\author{
JUNKICHI YOKOYAMA ${ }^{1,2}$, YASUHISA HASEGAWA ${ }^{3}$, MASASHI SUGASAWA ${ }^{4}$, \\ AKIHIRO SHIOTANI $^{5}$, YOSHIKO MURAKAMI ${ }^{6}$, SHINICHI OHBA ${ }^{7}$ and NAOYUKI KOHNO ${ }^{1}$ \\ ${ }^{1}$ Department of Otolaryngology-Head and Neck Surgery, Kyorin University School of Medicine, Tokyo 181-8611; \\ ${ }^{2}$ Department of Otolaryngology-Head and Neck Surgery, Nadogaya Hospital, Chiba 277-0084, ${ }^{3}$ Department of Head and \\ Neck Surgery, Aichi Cancer Center Hospital, Nagoya, Aichi 464-8681; ${ }^{4}$ Department of Otolaryngology-Head and \\ Neck Surgery, Saitama Medical University International Medical Center, Saitama 350-1298; ${ }^{5}$ Department of \\ Otolaryngology-Head and Neck Surgery, National Defence Medical College, Tokorozawa, Saitama 359-8513; \\ ${ }^{6}$ Department of Pathological Diagnosis, Nagoya Medical Center, Nagoya, Aichi 460-0001; ${ }^{7}$ Department of \\ Otolaryngology-Head and Neck Surgery, Juntendo University School of Medicine, Tokyo 113-8341, Japan
}

Received March 8, 2020; Accepted July 17, 2020

DOI: $10.3892 / \mathrm{mco} .2020 .2111$

\begin{abstract}
In the current study, the utility of sentinel node (SN) identification using indocyanine green (ICG) was investigated for oral cancers in the clinical N0 stage. The current study was a prospective, multicentre, phase II clinical trial that was conducted in Japan. A total of 18 patients were included. Before surgery, the patients underwent lymphoscintigraphy to map the SNs. During surgery, radioactive isotope (RI) mapping was used to detect the SNs, and ICG was subsequently injected. ICG mapping of the SNs was then performed through the skin. The primary tumour was resected, and a neck flap was elevated for neck dissection, followed by SN biopsy (SNB) using RI or ICG mapping. With the RI method, a total of 63 SNs were detected. Among these SNs, 8 (12.7\%) were positive for metastasis, including those with isolated tumour cells (ITCs). The median number of SNs per patient identified by SNB was 4. With the ICG method, a total of 67 SNs were detected. Among these SNs, 7 (10.4\%) were positive for metastasis, including those with ITCs. The median number of SNs per patient identified by SNB was 4 (range, 1-6). The 5 -year overall survival (OS) of all patients was $83.3 \%$, and the 5-year disease-free survival (DFS) of all patients was $76.7 \%$. The neck compression technique is a simple method that can be used to facilitate surgical procedures of ICG fluorescence navigated SNB for head and neck cancer.
\end{abstract}

Correspondence to: Dr Junkichi Yokoyama, Department of Otolaryngology-Head and Neck Surgery, Kyorin University School of Medicine, 6-20-2 Shinkawa, Mitaka-shi, Tokyo 181-8611, Japan E-mail: jyokoyama2@yahoo.co.jp

Key words: indocyanine green, near-infrared fluorescence, sentinel node, head and neck cancer, minimally invasive treatment

\section{Introduction}

Occult lymph node (LN) metastases, which are clinically negative nodes, are estimated to exist in $20-30 \%$ of oral cavity cancers. For T1 and T2 oral cancers, there are two surgical treatment strategies $(1,2)$. In some patients in which the 'wait and see' strategy is employed, unnecessary surgery can be avoided. However, delayed neck metastases can occur and occasionally develops into advanced stages. To avoid recurrence, selected neck dissection (ND) is often performed for high risk cases.

A comparative examination of these two surgical treatment strategies using a randomized trial (3) and meta-analysis (4) revealed a prognostic advantage of selective ND.

Selective neck dissection at the time of primary surgery revealed no metastases to the neck in approximately $70 \%$ of patients and consequently, ND appears to be an unnecessary surgical procedure for most patients. This possibility of patients undergoing unnecessary surgery reaffirms the importance of performing cervical LN staging before treatment. Therefore, the development of a new staging system with high reliability that extends beyond conventional diagnostic imaging is necessary.

The effectiveness of SNB as an accurate staging tool has been demonstrated $(2,5)$. To date, sentinel node ( $\mathrm{SN}$ ) biopsies have required radioactive isotopes (RIs). If an $\mathrm{SN}$ in the head and neck region could be identified using the fluorescence properties of indocyanine green (ICG), it would be possible to overcome the issues of radiation exposure for medical personnel and patients, institutional limitations, and the complex procedures that arise from the conventional use of RIs as tracers. This new method could be performed at any institution without radiation exposure, and we expect that this method would benefit patients.

We designed the present study to examine the usefulness of the transcutaneous neck compression technique and the diagnostic accuracy of ICG fluorescence navigated SNBs in comparison to the RI method. 
The ICG method as a non-RI method is convenient and widely applicable and may be useful for the diagnosis and treatment of the early stages not only in oral cancer, but also head and neck cancer.

\section{Materials and methods}

Patients. This study was a prospective, multicentre, phase II clinical trial (UMIN00006509) conducted in Japan. Between November 2011 and November 2012, 20 patients from 3 medical facilities were enrolled. Patients were included if they had been diagnosed with $\mathrm{cN} 0$ stage disease, had a pathologically confirmed squamous cell carcinoma, and had a tumour in the clinical 'late-T2' stage (i.e., a T2 tumour with a diameter $\geq 3 \mathrm{~cm}$ or any $\mathrm{T} 2$ tumour with a tumour depth $\geq 5 \mathrm{~mm}$ ) or T3 stage. The clinical stage was determined by physical examination and radiological computed tomography (CT). The 7th UICC classification was adopted in this study. Tumour depth was defined as the distance from the surrounding normal tissue to the bottom of the tumour and was estimated using CT or magnetic resonance imaging (MRI) in addition to palpation. The procedures used in this study were approved by the institutional review boards of each institution, and written consent was obtained from all the patients.

Study procedures. A flow diagram of the procedures used in this study is presented in Fig. 1. Before surgery, the enrolled patients underwent lymphoscintigraphy to map the SNs. During surgery, the SNs were initially detected via RI mapping, and ICG was subsequently injected. Next, ICG mapping of the SNs was performed through the skin. Skin marking was done by ICG mapping method.

The primary tumour was resected, a neck flap was elevated for neck dissection, and SN biopsy was performed using RI and ICG mapping. Selective ND with SN basin dissection was performed according to the SN status (6). In cases of pull-through resection, the SN biopsy was performed before the resection. The patients with positive LNs but no extranodal extension (ENE) were carefully followed up after surgery. However, if ENE was observed, radiotherapy with/without chemotherapy was administered. Chemoradiotherapy was applied to patients with multiple neck metastasis (N2b) and ENE.

RI SN mapping. Preoperatively, the SNs were localized using conventional lymphoscintigraphy. In some cases, single-photon emission CT with CT (SPECT/CT) was performed to obtain additional information. Technetium $99 \mathrm{~m}\left({ }^{99 \mathrm{~m}} \mathrm{Tc}\right)$ phytate (FUJIFILM RI Pharma Co., Ltd. and Nihon Medi-Physics Co., Ltd.), which was used as the radiotracer, was injected submucosally ( $74 \mathrm{MBq}$ in $1 \mathrm{ml}$ ) $24 \mathrm{~h}$ before surgery at 4 points (one point in each quadrant) around the primary tumour.

During surgery, radioactive SNs were detected with handheld gamma probes, a neo2000 probe (Neoprobe) or a Navigator ${ }^{\mathrm{TM}}$ GPS probe (RMD Instruments).

ICGfluorescence SN mapping. An infrared fluorescence imaging system (Photodynamic Eye, Hamamatsu Photonics, Japan) that consisted of light-emitting diodes (LED) set at $760 \mathrm{~nm}$ was used as the light source, and a charge-coupled device camera with a cut filter set below $820 \mathrm{~nm}$ was used as a detector to obtain near-infrared (NIR) fluorescence images. ICG (5 mg/2 ml) was injected submucosally at 4 points around the primary tumour intraoperatively. Several minutes were usually required after ICG injection until adequate amounts of ICG accumulated in the SNs. After $10 \mathrm{~min}$, transcutaneous detection of the SNs was initiated by performing the neck compression technique. When the neck skin was compressed against the posterior neck or oral floor with a transparent plastic cone device (Hamamatsu Photonics) (Fig. 2), a fluorescence signal appeared at the bottom of the device because the LN moved close to the surface (7). The LNs appeared as round areas of intense signal.

The lymphatic vessels of the oral cavity and the laryngopharynx drain directly into the deep nodes (i.e., the submental, submandibular and jugular nodes). When the ICG was injected submucosally, the subcutaneous lymphatic vessels did not exhibit a fluorescent signal.

After neck flap elevation for neck dissection, most of the ICG-mapped SNs were detected without the neck compression technique. RI- and/or ICG-mapped SNs were biopsied using a gamma probe or a fluorescence imaging system.

Because ICG is not a colloid, it spreads to the secondary LNs over time. Therefore, at biopsy, LNs other than the SN also emitted weak fluorescence. Numbers of SNs are maximally limited to six. Skin marking was used to ensure the exact locations of the SNs to be extracted; these SNs exhibited strong fluorescence intensities.

Histopathological evaluation. All the SNs were subjected to intraoperative pathological evaluation. All the other LNs, including the non-radioactive and non-fluorescent LNs, were considered non-SNs. The non-SNs were divided longitudinally into 2 specimens, and a single representative cross section was stained with haematoxylin-eosin (HE) for a final postoperative diagnosis. The SNs were cut into $2-\mathrm{mm}$ blocks, and $4-\mu \mathrm{m}$ sections from each block were used for intraoperative frozen section analysis. Additional sections were stained with HE and the cytokeratinAE1/AE3 (Agilent Technologies) stain for a final postoperative diagnosis.

Occult metastases were classified into 3 categories (8): Isolated tumour cells (ITCs; $<0.2 \mathrm{~mm}$ in diameter), micrometastases $(0.2-2 \mathrm{~mm})$, and macrometastases $(>2 \mathrm{~mm})$. Macrometastasis and micrometastasis were considered indicative of pathological positivity for LN metastasis but ITCs were not.

Statistical analyses. The primary endpoint of this study was the SN identification rate of the ICG fluorescence method (cases with the ICG method/cases with the RI method). The $\mathrm{SN}$ identification rates are evaluated by the concordance rates between the RI and ICG methods.

The assumed SN identification rate of the ICG mapping was $95 \%$, the threshold identification rate was $70 \%$, the $\alpha$ error was 0.05 , the detection power was 0.80 , and the required number of cases was 15 .

Considering the possibility of some unevaluable cases, 18 was the target number of cases.

Kaplan-Meier survival curves were used to analyse the survival outcomes. We defined overall survival (OS) as the duration between the date of registration and the date of death or last follow-up. Disease-free survival (DFS) was defined 


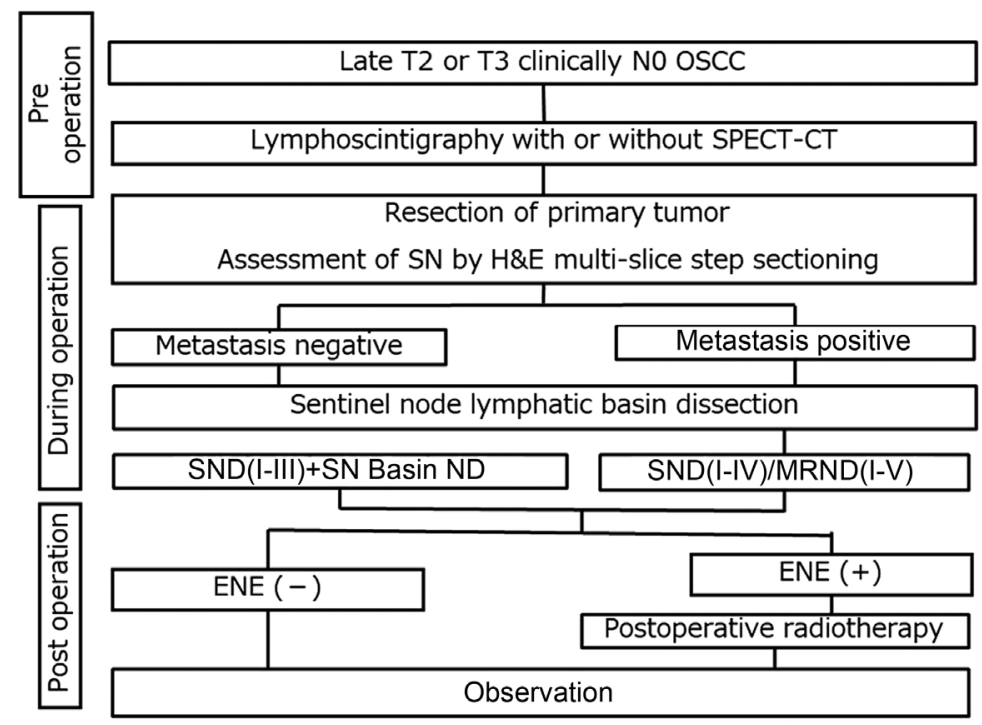

Figure 1. Flow diagram of the procedures used in this study. OSCC, oral squamous cell carcinoma; SPECT, single-photon emission computed tomography; SN, sentinel node; SND, selective neck dissection; ENE, extranodal extension; MRND, modified radical neck dissection.
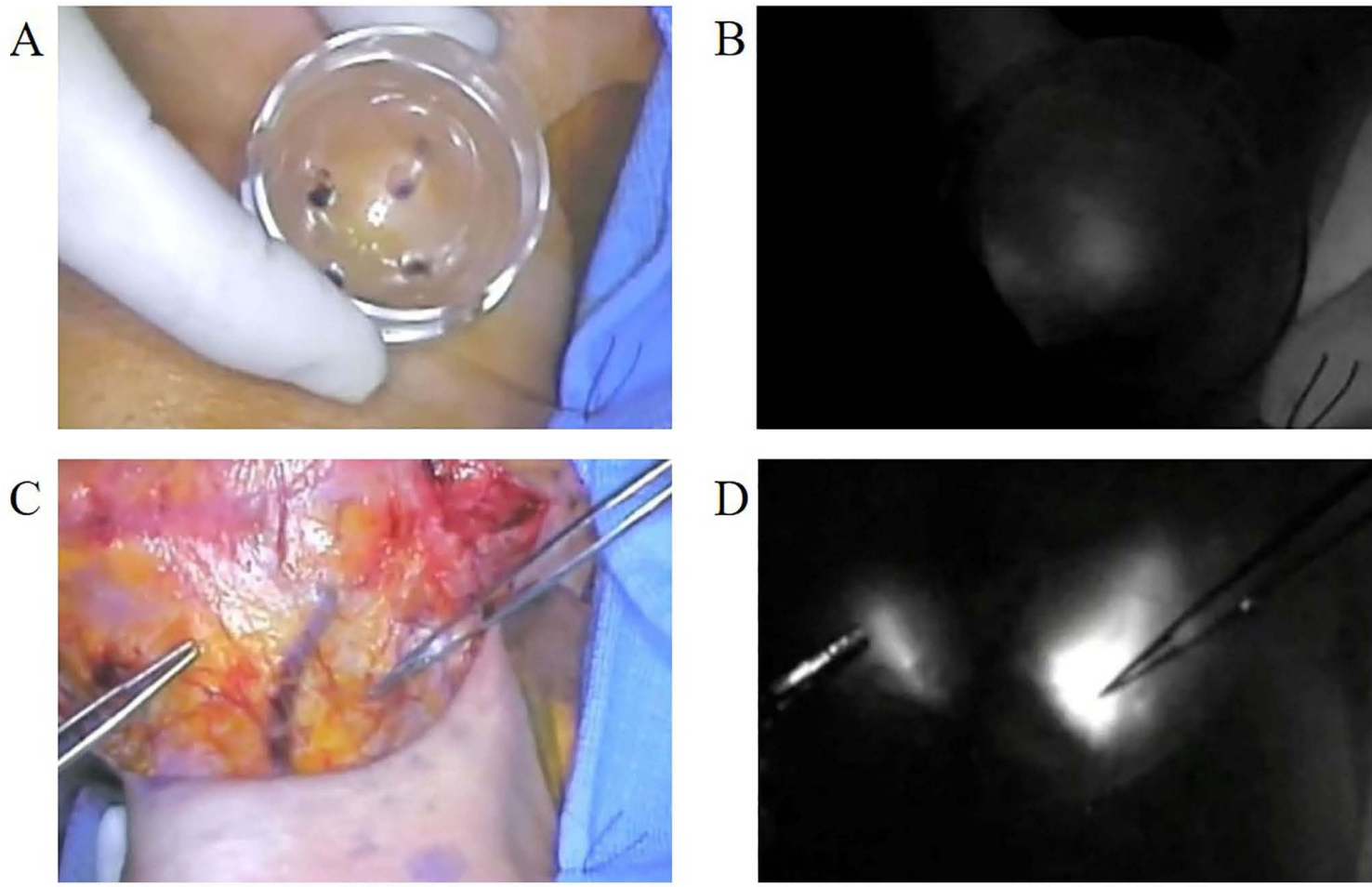

Figure 2. ICG fluorescence SN mapping. An infrared fluorescence imaging system, plastic cones, and the neck compression technique. The neck compression technique using a (A) plastic cone can be applied to confirm (B) deep fluorescent SNs. After (C) skin elevation, the SNs were visualized (D) without neck compression. SN, sentinel node; ICG, indocyanine green.

as the duration between the date of registration and the date of relapse, second primary tumour, death, or last follow-up. $\mathrm{P}<0.05$ was considered to indicate a statistically significant difference. All the statistical analyses were performed using EZR (v1.32) (9) on R commander.

\section{Results}

Subject characteristics. From July 2011 to July 2012, 20 patients were enrolled in the study. Of these, 2 patients were excluded because of final preoperative diagnoses of positive nodes. SNs were identified in all 18 enrolled patients. The median follow-up period was 62.1 months (range: 52.3-72.6 months). The patient characteristics are summarized in Table $\mathrm{I}$.

Primary endpoint. The SN identification concordance rates between the RI and ICG methods are presented in Table II. The concordance rate of the ICG and RI methods at the case level was $94 \%$ (17/18, 95\% CI: 0.73-1.00), and the concordance 
rate for the overall RI and ICG methods was $72 \%$ (13/18, 95\% CI: 0.47-0.90); thus, the agreement rates were high.

The SN identification concordance rates of the ICG and RI methods and the RI and ICG methods at the LN level were 97\% (61/63) and 91\% (61/67), respectively. (Table II); thus, the agreement rates were high. These results indicate that the primary endpoint goal was achieved.

Distributions and metastatic statuses of the SNs. The detailed distributions and metastatic statuses of the SNs are illustrated in Figs. 3 and 4. With the RI method, a total of 63 SNs were detected (18 level I, 26 level II, 8 level III, 3 level IV, and 8 SNs in the contralateral region of the neck). Among these SNs, 8 $(12.7 \%)$ were positive for metastasis, including ITCs ( 2 were level I, 4 were level II, 1 was level III, 0 were level V and 1 was in the contralateral region of the neck; Fig. 3). The median number of SNs per patient identified by SNB was 4 (range: 2-6).

With the ICG method, a total of 67 SNs were detected (20 were level I, 24 were level II, 8 were level III, 3 were level IV, and 12 were in the contralateral region of the neck). Among these SNs, 7 (10.4\%) were positive for metastasis, including ITCs ( 2 were level I, 3 were level II, 1 was level III, 0 were level $\mathrm{V}$ and 1 was in the contralateral region of the neck; Fig. 4). The median number of SNs per patient identified by SNB was 4 (range: 1-6).

Outcomes. There was 1 case of ENE, which was treated with postoperative chemoradiotherapy. Five patients experienced recurrences or second primary tumours during the follow-up period, 3 of which occurred at the primary site, 1 of which occurred in the neck, and 1 of which occurred at a second primary site.

The cervical recurrence was a case of $\mathrm{pN} 2 \mathrm{~b}$, which was from the dissected area of the affected neck.

Of the 3 patients who experienced recurrence at the primary site, 2 had suspicious multicentric cancer.

The 5-year OS of all the patients was $83.3 \%$ (95\% CI: 0.57-0.88; Fig. 5), and the 5-year DFS of all the patients was 76.7\% (95\% CI: 0.49-0.91; Fig. 6).

\section{Discussion}

The results of a phase III study (3) in India demonstrated a prognostic advantage of selective neck dissection (SND) for early oral cancer in 2015. However, the surgical indication for SND could be limited if the diagnosis of NO cases or the regular follow-up of patients could be strictly performed.

If SND is performed for all N0 cases of early cancer, unnecessary surgery will be performed for more than $70 \%$ of patients, and surgery occasionally causes postoperative dysfunction or complications. In this respect, SN biopsy has been demonstrated to be less invasive than SND (10-12). Moreover, avoiding unnecessary procedures is expected to reduce medical expenses (13-15). Furthermore, potential metastasis to level IV, which occurs outside the range of supraomohyoid neck dissection (SOND), has been reported to occur in $9-10 \%$ of cases (16-18). This missed metastasis is also a problem for improving survival. SN biopsy is considered a useful solution to these problems.
Table I. Patients characteristics.

\begin{tabular}{|c|c|c|}
\hline Characteristics & Number & $\%$ \\
\hline Age, median (range), years & $54.5(26-82)$ & \\
\hline \multicolumn{3}{|l|}{ Sex } \\
\hline Male & 11 & 61 \\
\hline Female & 7 & 39 \\
\hline \multicolumn{3}{|l|}{ Tumor location } \\
\hline Tongue & 15 & 83 \\
\hline Gingiva & 2 & 11 \\
\hline \multicolumn{3}{|l|}{ Clinical T stage $\mathrm{e}^{\mathrm{a}}$} \\
\hline Late $\mathrm{T} 2$ & 17 & 94 \\
\hline $\mathrm{T} 3$ & 1 & 6 \\
\hline \multicolumn{3}{|l|}{ Pathologic TN stage $(\mathrm{pT})$} \\
\hline Tis & 1 & 6 \\
\hline $\mathrm{T} 1$ & 11 & 61 \\
\hline $\mathrm{T} 2$ & 3 & 17 \\
\hline $\mathrm{T} 3$ & 1 & 6 \\
\hline $\mathrm{T} 4 \mathrm{a}$ & 2 & 11 \\
\hline \multicolumn{3}{|l|}{ Pathologic TN stage $(\mathrm{pN})$} \\
\hline No & 12 & 67 \\
\hline N1 & 4 & 22 \\
\hline $\mathrm{N} 2 \mathrm{~b}$ & 2 & 11 \\
\hline Positive sentinel nodes (cases) & 6 & 33 \\
\hline Positive sentinel nodes (nodes) & 8 & \\
\hline ITC & 1 & 13 \\
\hline $0.2-2 \mathrm{~mm}$ & 2 & 25 \\
\hline$<2 \mathrm{~mm}$ & 5 & 63 \\
\hline \multicolumn{3}{|l|}{ Tumor resection method } \\
\hline Trans-oral/cervical & 14 & 78 \\
\hline Pull-through & 4 & 22 \\
\hline \multicolumn{3}{|l|}{ Reconstruction method } \\
\hline Primary suture & 14 & 78 \\
\hline Flap & 4 & 22 \\
\hline \multicolumn{3}{|l|}{ Node dissection } \\
\hline Ipsilateral & 11 & 61 \\
\hline Bilateral & 6 & 33 \\
\hline Sentinel node biopsy & 1 & 6 \\
\hline Adjuvant chemoradiotherapy & 1 & 6 \\
\hline
\end{tabular}

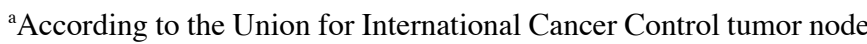
metastasis staging system (7th edition); ITC, isolated tumour cells.

SND and SN biopsy have been studied in early-stage oral cancer and also in early pharyngeal and laryngeal cancers with the transoral approach.

In an investigation of $40 \mathrm{NO}$ laryngopharyngeal carcinoma patients who underwent transoral resection, the author indicated that the indications for SND include a tumour depth exceeding $1 \mathrm{~mm}$ and microvascular invasion. Because patients with tumour depths of 0.5-1.0 mm require sufficient observation, the author also indicated that these cases should be good candidates for SN biopsy (19). 
Table II. Concordance rate of RI and ICG mapping SNs.

\begin{tabular}{lllllllr}
\hline \multirow{2}{*}{ Detection methods } & \multicolumn{3}{c}{ Cases } & & \multicolumn{3}{c}{ Nodes } \\
\cline { 2 - 4 } \cline { 7 - 8 } & No. & $\%$ & $95 \% \mathrm{CI}$ & & No. & $\%$ & $95 \%$ CI \\
\hline ICG/RI & $17 / 18$ & 94 & $0.73-1.00$ & & $61 / 63$ & 97 & $0.89-1.00$ \\
RI/ICG & $13 / 18$ & 72 & $0.47-0.90$ & & $61 / 67$ & 91 & $0.82-0.97$ \\
\hline
\end{tabular}

CI, Confidence Interval; ICG, indocyanine green; RI, radioactive isotope; SN, sentinel nodes.

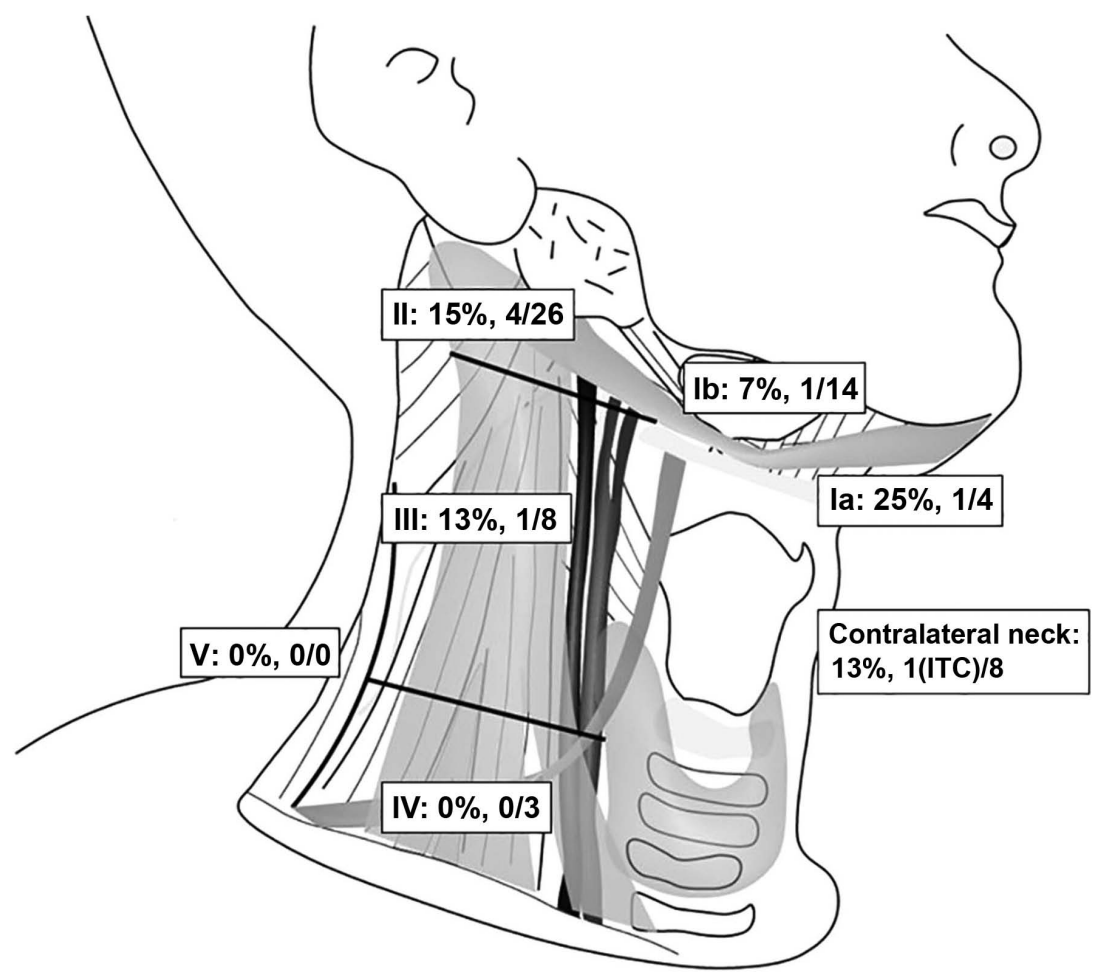

Figure 3. Distribution and metastatic status of the SNs. Number of detected SNs using RI mapping and positive nodes. SN, sentinel node; RI, radioactive isotope.

Need for an SN biopsy method and a non-RI method in throat cancer. In recent years, the transoral approach for pharyngo-laryngeal cancers has been reported to enable minimally invasive surgery for primary tumours. Additionally, this approach has the advantage of performing SN navigation neck dissection for occult $\mathrm{LN}$ metastasis while being even less invasive to the neck $(20,21)$. Future investigations are needed to verify whether the combination of primary tumour resection with a transoral approach and sentinel LN navigation neck dissection is possible.

When an RI method is applied for laryngeal or hypopharyngeal cancer, an endoscopic injection is needed, whereas a direct injection can be used for oropharyngeal cancer. This requirement limits the equality of medical care due to technical and equipment issues at institutions. Therefore, there is a need to develop non-RI methods for laryngeal cancers.

ICG fluorescence method. When SN biopsies are performed, RIs are conventionally used as tracers to identify the SNs. However, this method has the disadvantage of radiation exposure and thus requires improvement. ICG can be administered to the human body and is used as a reagent for examinations of the liver, ocular fundus, and other structures. ICG is excited by infrared light $(760-780 \mathrm{~nm})$ and emits near-infrared fluorescence at different wavelengths $(800-850 \mathrm{~nm})$ that are easily transmitted in the human body. The dynamic state can be observed non-invasively below the tissue surface using an infrared light detection camera following the injection of ICG into the body.

Because ICG accumulates in LNs, SNs can be visualized intraoperatively. Therefore, this method could be the best alternative to RI methods.

The ICG method has great advantages as a non-RI method, but two problems must be overcome. First, the depth of penetration of near-infrared light is limited, and second, the near-infrared signal spreads rapidly and diffusely because ICG is not a colloid. The longer the time between injection and detection, the more lymph nodes that are detected. It requires more skillful techniques because it requires detection in a short time. As more lymph nodes are detected in ICG, the number of SNs selected is approximately 4. In our study, out of several ICG infrared lymph nodes in level II, 2 bigger ICG 


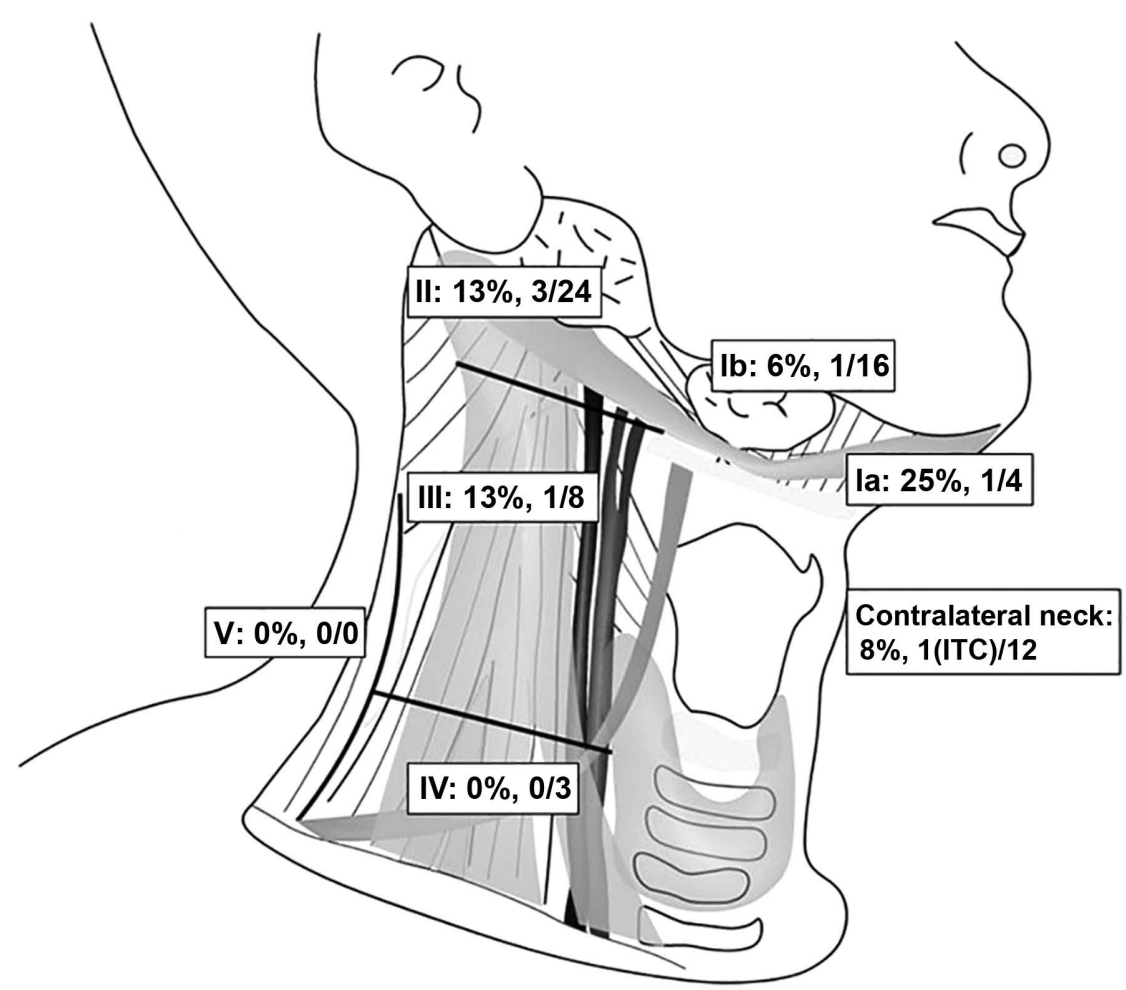

Figure 4. Distribution and metastatic status of the SNs. Number of detected SNs using ICG mapping and positive nodes. SN, sentinel node; ICG, indocyanine green.

infrared lymph nodes were incorrectly selected as SNs and a smaller metastatic node was not selected (Fig. 4).

ICG method also detected more SNs in the contralateral neck than RI method. It was estimated that this is why it depends on the duration from injection to biopsy.

The application of SN biopsy methods using ICG have been reported in breast cancer (22) and gastric cancer (23). However, Al-Dam et al (24) reported that the sensitivity and specificity of ICG in oral cavity cancer were $50 \%$ and $100 \%$, respectively. As ICG spreads quickly, the number of SLNs were usually more than RI colloid methods. However, the mean number of SLNs was 1.95 (1-3). The number of SLNs in 4 false negative cases were 1,1,2 and 3, respectively while in 4 positive cases they were 2, 3, 2 and 3, respectively. The mean number of SLNs of the two groups were 1.75 and 2.5, respectively. The number of SLNs were fewer than the average number 3.4 (25) and our cases (mean number 4). As it was difficult to select one or two metastatic SLNs from many fluorescence lymph nodes, the metastatic SLNs were probably not selected accurately as SLNs. van der Vorst et al (26), also reported that after injection of ICG with human serum albumin (complex: ICG:HSA), SLNs were observed to increase significantly over time. As SLNs increase significantly over time, it is necessary to set a limit on the number of SLNs needed. We recommend 3 or 4 SLNs in ICG method.

According to the eighth international symposium for SNB in head and neck cancer (8th SNB) in 2018, ICG fluorescence method has shown promise in identifying lymph nodes located close to the primary tumor (ie, FOM) that may otherwise be missed due to high gamma signal at the injection site (shine-through effect) (27). However, Schilling et al (28) recommend that ICG fluorescence method alone is not applicable for $\mathrm{SNB}$, yet support its use in combination with a radionuclide tracer. Murase et al (29), also reported that since using ICG fluorescent imaging and $99 \mathrm{~m}$-technetium-tin colloid were able to identify all positive SLNs, this combined method was useful for detecting SLNs in oral cavity cancer. However, ICG fluorescent imaging could not detect any SLNs transcutaneously. Compression method could facilitate the intraoperative localization of the SN in breast cancer (7). However, compression methods using a transparent plastic cone device have not been reported in oral cavity cancer. This is the first report describing the potential usefulness of compression methods using a transparent plastic cone device in oral cavity cancer.

In a basic experiment using the HyperEye Medical System (HEMS), the locations of cervical LNs could be visualized transcutaneously in rabbits. In pigs, which have thick skin and fat layers, the locations of LNs could not be identified transcutaneously. However, the LNs themselves emitted fluorescence, and it was possible to visualize the LNs based on accumulated ICG in the fat layer (30).

NIR fluorescence penetrates human tissues to a depth of 1-2 cm and allows for the transcutaneous visualization of SLNs and lymphatic vessels (31). Usually, NIR fluorescence does not reach the jugular nodes. Nakamura reported that fluorescent SNs could be visualized transcutaneously with the HEMS camera when the skin over the SN received finger pressure in 6 head and neck cancers (32). The jugular nodes are the frequent targets of metastasis in head and neck cancer. As the jugular nodes are located in the deep neck and behind the sternocleidomastoid muscle of the neck, it is difficult to confirm the SNs through the skin. Fortunately, there are some neck spaces without muscles including carotid triangle, submental triangle, and lessor supraclaviclar fossa in the neck. The neck compression technique using a plastic cone can be applied to confirm deep SNs. As a result, we can definitely detect lymphnodes through these spaces by using a plastic cone. 


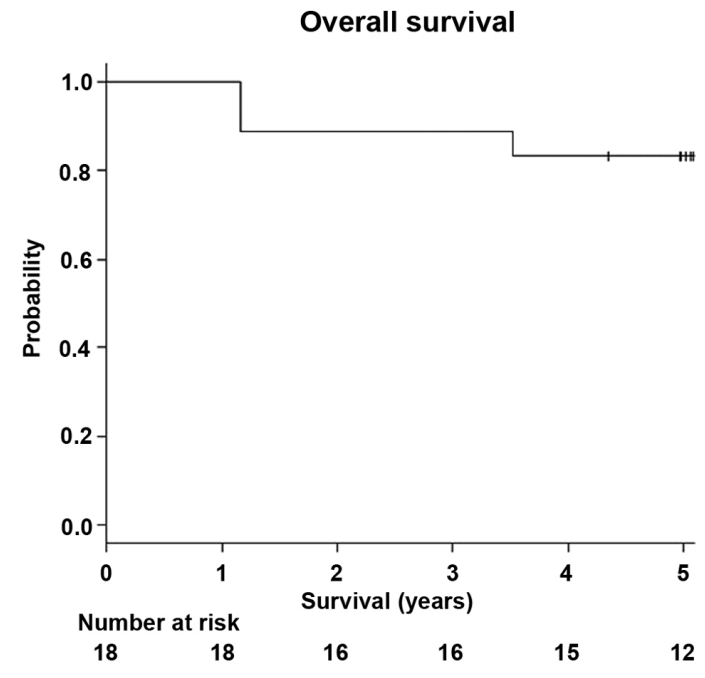

Figure 5. Kaplan-Meier curves of OS. OS, overall survival.

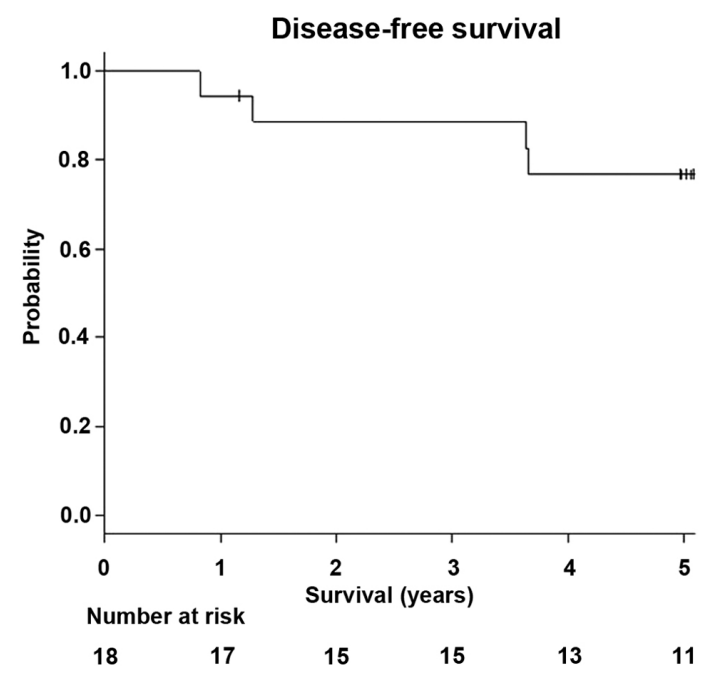

Figure 6. Kaplan-Meier curves of DFS. DFS, disease-free survival.

As previously mentioned, one of the major drawbacks of this method is the rapid migration of ICG through the lymphatic system, which limits the diagnostic window and can lead to the undesirable detection of downstream nodes. The exact detection of SNs with ICG mapping requires a sufficient learning curve. Araki et al (33), resolved this problem by mixing ICG with phytate colloid to retard its migration and demonstrated the feasibility of this technique in a nude mouse study.

This prospective multicenter study has some limitations such as the learning curve associated with operative procedures. Before conducting this study, we held meetings in order to establish uniform surgical techniques and to shorten the learning curve. Inexperienced operators went to study SNB using ICG fluorescence method with a plastic cone device at experienced surgical centers. Furthermore, in the first trials conducted at institutions with limited experience, skilled surgeons went to instruct inexperienced surgeons and to promote uniform accessibility of SNB using the ICG fluorescence method.
As previously mentioned, the ICG method has two problems associated with the limited depth of penetration of near-infrared light and the rapid spread of ICG. By compression through several neck spaces with a plastic cone device, the jugular nodes and submandibular nodes can be positively detected transcutaneously. As SLNs increase over time due to spreading ICG, there need to be limitations in the number of SLNs. Before conducting this prospective, multicentre, phase II clinical trial, we discussed and practiced detecting transcutaneously SLNs by ICG fluorescence method with a plastic cone device. As detecting rapidly spreading near-infrared lymph nodes, we decided to set the number of SLNs at approximately 4.

As overcoming these two problems by using ICG fluorescence method with a plastic cone device, we demonstrated the high concordance between RI and ICG mapping of SNs in oral cavity cancer. As a result, we expect ICG fluorescence method with a plastic cone can be employed as an alternative technique to SN mapping in oral cavity cancer in near future.

The neck compression technique demonstrated high concordance between RI and ICG mapping of SNs in oral cavity cancer. This simple method can facilitate the surgical procedures of ICG fluorescence-navigated SNB transcutaneously in oral cavity cancer.

\section{Acknowledgements}

Not applicable.

\section{Funding}

The present study was supported by a Health and Labour Sciences Research Grant for Clinical Cancer Research (grant nos. H21-Gannrinshou-Ippan-016 and H24-Gannrinshou-Ippan-006) from the Ministry of Health, Labour and Welfare in Japan. The present study was also supported by KAKENHI (Grants-in-Aid for Scientific Research) 16K11247 from the Ministry of Education, Culture, Sports, Science and Technology (MEXT).

\section{Availability of data and materials}

The datasets during and/or analyzed during the present study available from the corresponding author on reasonable request.

\section{Authors' contributions}

YH and JY designed the study. AS, MS and SO contribute to acquisition of data. JY and YH drafted the manuscript. MS and NK performed the statistical analysis. YM conducted the pathological diagnosis. JY and YH revised the manuscript. All authors read and approved the final manuscript.

\section{Ethics approval and consent to participate}

The current study was approved by the Ethics Committee of each School and Hospital of Head and Neck Surgery and registered in the prospective, multicentre, phase II clinical trial (UMIN00006509) in Japan. All patients provided written informed consent before the study. 


\section{Patient consent for publication}

Written informed consent for the publication of any associated data was obtained from all patients.

\section{Competing interests}

The authors declare that they have no competing interests.

\section{References}

1. Den Toom IJ, Heuveling DA, Flach GB, van Weert S, Karagozoglu KH, van Schie A, Bloemena E, Leemans CR and de Bree R: Sentinel node biopsy for early-stage oral cavity cancer: The VU university medical center experience. Head Neck 37: 573-578, 2015

2. Schilling C, Stoeckli SJ, Haerle SK, Broglie MA, Huber GF, Sorensen JA, Bakholdt V, Krogdahl A, von Buchwald C, Bilde A, et al: Sentinel European node trial (SENT): 3-year results of sentinel node biopsy in oral cancer. Eur J Cancer 51: 2777-2784, 2015

3. D'Cruz AK, Vaish R, Kapre N, Dandekar M, Gupta S, Hawaldar R, Agarwal JP, Pantvaidya G, Chaukar D, Deshmukh A, et al: Elective versus therapeutic neck dissection in node-negative oral cancer. N Engl J Med 373: 521-529, 2015.

4. Abu-Ghanem S, Yehuda M, Carmel NN, Leshno M, Abergel A, Gutfeld $\mathrm{O}$ and Fliss DM: Elective neck dissection vs observation in early-stage squamous cell carcinoma of the oral tongue with no clinically apparent lymph node metastasis in the neck: A systematic review and meta-analysis. JAMA Otolaryngol Head Neck Surg 142: 857-865, 2016.

5. Civantos FJ, Zitsch RP, Schuller DE, Agrawal A, Smith RB Nason R, Petruzelli G, Gourin CG, Wong RJ, Ferris RL, et al: Sentinel lymph node biopsy accurately stages the regional lymph nodes for T1-T2 oral squamous cell carcinomas: Results of a prospective multi-institutional trial. J Clin Oncol 28: 1395-400, 2010.

6. Miura K, Hirakawa H, Uemura H, Yoshimoto S, Shiotani A, Sugasawa M, Homma A, Yokoyama J, Tsukahara K, Yoshizaki T, et al: Sentinel node biopsy for oral cancer: A prospective multicenter phase II trial. Auris Nasus Larynx 44: 319-326, 2017.

7. Kitai $\mathrm{T}$ and Kawashima M: Transcutaneous detection and direct approach to the sentinel node using axillary compression technique in ICG fluorescence-navigated sentinel node biopsy for breast cancer. Breast Cancer 19: 343-348, 2012.

8. Hermanek P, -Hutter RV, Sobin LH and Wittekind C: International union against cancer. Classification of isolated tumor cells and micrometastasis. Cancer 86: 2668-2673, 1999.

9. Kanda Y: Investigation of the freely available easy-to-use software 'EZR' for medical statistics. Bone Marrow Transplant 48: 452-458, 2013

10. Schiefke F, Akdemir M, Weber A, Akdemir D, Singer S and Frerich B: Function, postoperative morbidity, and quality of life after cervical sentinel node biopsy and after selective neck dissection. Head Neck 31: 503-512, 2009.

11. Murer K, Huber GF, Haile SR and Stoeckli SJ: Comparison of morbidity between sentinel node biopsy and elective neck dissection for treatment of the n0 neck in patients with oral squamous cell carcinoma. Head Neck 33: 1260-1264, 2011.

12. Hernando J, Villarreal P, Alvarez-Marcos F, Gallego L, García-Consuegra L and Junquera L: Comparison of related complications: Sentinel node biopsy versus elective neck dissection. Int J Oral Maxillofac Surg 43: 1307-1312, 2014.

13. Govers TM, Takes RP, Baris Karakullukcu M, Hannink G, Merkx MA, Grutters JP and Rovers MM: Management of the NO neck in early stage oral squamous cell cancer: A modeling study of the cost-effectiveness. Oral Oncol 49: 771-777, 2013.

14. Kosuda S, Kusano S, Kohno N, Ohno Y, Tanabe T, Kitahara S and Tamai S: Feasibility and cost-effectiveness of sentinel lymph node radiolocalization in stage N0 head and neck cancer. Arch Otolaryngol Head Neck Surg 129: 1105-1109, 2003.

15. O'Connor R, Pezier T, Schilling $\mathrm{C}$ and McGurk M: The relative cost of sentinel lymph node biopsy in early oral cancer. J Craniomaxillofac Surg 41: 721-727, 2013.
16. Shah JP: Patterns of cervical lymph node metastasis from squamous carcinomas of the upper aerodigestive tract. Am J Surg 160: 405-409, 1990

17. Byers RM, Weber RS, Andrews T, McGill D, Kare R and Wolf P: Frequency and therapeutic implications of 'skip metastases' in the neck from squamous carcinoma of the oral tongue. Head Neck 19: 14-19, 1997.

18. Crean SJ, Hoffman A, Potts J and Fardy MJ: Reduction of occult metastatic disease by extension of the supraomohyoid neck dissection to include level IV. Head Neck 25: 758-762, 2003.

19. Tomifuji M, Imanishi Y, Araki K, Yamashita T, Yamamoto S, Kameyama K and Shiotani A: Tumor depth as a predictor of lymph node metastasis of supraglottic and hypopharyngeal cancers. Ann Surg Oncol 18: 490-496, 2011.

20. Shiotani A, Tomifuji M, Araki K, Yamashita T and Saito K: Videolaryngoscopic transoral en bloc resection of supraglottic and hypopharyngeal cancers using laparoscopic surgical instruments. Ann Otol Rhinol Laryngol 119: 225-232, 2010.

21. Yamashita T, Tomifuji M, Araki K, Kurioka T and Shiotani A: Endoscopic transoral oropharyngectomy using laparoscopic surgical instruments. Head Neck 33: 1315-1321, 2011.

22. Tagaya N, Aoyagi H, Nakagawa A, Abe A, Iwasaki Y, Tachibana M and Kubota K: A novel approach for sentinel lymph node identification using fluorescence imaging and image overlay navigation surgery in patients with breast cancer. World J Surg 35: 154-158, 2011.

23. Tajima Y, Murakami M, Yamazaki K, Masuda Y, Kato M, Sato A, Goto S, Otsuka K, Kato T and Kusano M: Sentinel node mapping guided by indocyanine green fluorescence imaging during laparoscopic surgery in gastric cancer. Ann Surg Oncol 17: 1787-1793, 2010.

24. Al-Dam A, Precht C, Barbe A, Kohlmeier C, Hanken H, Wikner J, Schön G, Heiland M and Assaf AT: Sensitivity and specificity of sentinel lymph node biopsy in patients with oral squamous cell carcinomas using indocyanine green fluorescence imaging. J Craniomaxillofac Surg 46: 1379-1384, 2018.

25. Peng H, Wang SJ, Niu X, Yang X, Chi C and Zhang G: Sentinel node biopsy using indocyanine green in oral/oropharyngeal cancer. World J Surg Oncol 17: 278, 2015.

26. van der Vorst JR, Schaafsma BE, Verbeek FP, Keereweer S, Jansen JC, van der Velden LA, Langeveld AP, Hutteman M, Löwik CW, van de Velde CJ, et al: Near-infrared fluorescence sentinel lymph node mapping of the oral cavity in head and neck cancer patients. Oral Oncol 49: 15-19, 2013.

27. Christensen A, Juhl K, Charabi B, Mortensen J, Kiss K, Kjær A and von Buchwald C: Feasibility of real-time near-infrared fluorescence tracer imaging in sentinel node biopsy for oral cavity cancer patients. Ann Surg Oncol 23: 565-572, 2016.

28. Schilling C, Stoeckli SJ, Vigili MG, de Bree R, Lai SY, Alvarez J, Christensen A, Cognetti DM, D'Cruz AK, Frerich B, et al: Surgical consensus guidelines on sentinel node biopsy (SNB) in patients with oral cancer. Head Neck 41: 2655-2664, 2019.

29. Murase R, Tanaka H, Hamakawa T, Goda H, Tano T, Ishikawa A, Hino S, Sumida T, Nakashiro K and Hamakawa H: Double sentinel lymph node mapping with indocyanine green and $99 \mathrm{~m}$-technetium-tin colloid in oral squamous cell carcinoma. Int J Oral Maxillofac Surg 44: 1212-1217, 2015.

30. Yamauchi K, Nagafuji H, Nakamura T, Sato T and Kohno N: Feasibility of ICG fluorescence-guided sentinel node biopsy in animal models using the hypereye medical system. Ann Surg Oncol 18: 2042-2047, 2011.

31. Fujisawa Y, Nakamura Y, Kawachi Y and Otsuka F: Indocyanine green fluorescence-navigated sentinel node biopsy showed higher sensitivity than the radioisotope or blue dye method, which may help to reduce false-negative cases in skin cancer. J Surg Oncol 106: 41-45, 2012

32. Nakamura T, Kogashiwa Y, Nagafuji H, Yamauchi K and Kohno N: Validity of sentinel lymph node biopsy by ICG fluorescence for early head and neck cancer. Anticancer Res 35: 1669-1674, 2015.

33. Araki K, Mizokami D, Tomifuji M, Yamashita T, Ohnuki K, Umeda IO, Fujii H, Kosuda S and Shiotani A: Novel indocyanine green-phytate colloid technique for sentinel node detection in head and neck: Mouse study. Otolaryngol Head Neck Surg 151: 279-285, 2014. Attribution-NonCommercial-NoDerivatives 4.0 International (CC BY-NC-ND 4.0) License. 